\title{
Posiphen as a candidate drug to lower CSF amyloid precursor protein, amyloid- $\beta$ peptide and $\tau$ levels: target engagement, tolerability and pharmacokinetics in humans
}

\author{
Maria L Maccecchini, ${ }^{1}$ Mee Young Chang, ${ }^{1}$ Catherine Pan, ${ }^{2}$ Varghese John, ${ }^{3}$ \\ Henrik Zetterberg, ${ }^{4}$ Nigel $\mathrm{H}$ Greig ${ }^{5}$
}

- Additional materials are published online only. To view these files please visit the journal online (http://jnnp.bmj. com/content/83/9.toc).

${ }^{1}$ QR Pharma, Inc., Berwyn, Pennsylvania, USA

${ }^{2}$ Inarian Neurodiagnostics, LLC., Mercer Island, Washington, USA

${ }^{3}$ Alzheimer's Drug Discovery Network, Buck Institute for Research on Aging, Novato, California, USA

${ }^{4}$ Clinical Neurochemistry Laboratory, Sahlgrenska University, Hospital/ Mölndal, Mölndal, Sweden

${ }^{5}$ Drug Design \& Development Section, Laboratory of Neurosciences, Intramural Research Program, National Institute on Aging, National Institutes of Health, Baltimore, Maryland, USA

\section{Correspondence to}

Dr Nigel H Greig, Drug Design \& Development Section, Laboratory of Neurosciences, Intramural Research Program, National Institute on Aging, National Institutes of Health, 251 Bayview Blvd., Room 05C220, Baltimore, Maryland 21224, USA;

greign@grc.nia.nih.gov

Maria Maccecchini; maccecchini@qrpharma.com

Received 23 February 2012 Revised 21 May 2012 Accepted 23 May 2012 Published Online First 11 July 2012

This paper is freely available online under the BMJ Journals unlocked scheme, see http:// jnnp.bmj.com/site/about/ unlocked.xhtml

\section{ABSTRACT}

Aim A first in human study to evaluate tolerability and pharmacokinetics followed by an early proof of mechanism (POM) study to determine whether the small orally, available molecule, Posiphen tartrate (Posiphen), lowers secreted ( $s$ ) amyloid- $\beta$ precursor protein (APP) $\alpha$ and $-\beta$, amyloid- $\beta$ peptide $(A \beta)$, tau $(\tau)$ and inflammatory markers in CSF of patients with mild cognitive impairment (MCI).

Study design Posiphen single and multiple ascending dose phase 1 randomised, double blind, placebocontrolled safety, tolerance, pharmacokinetic studies were undertaken in a total of 120 healthy volunteers to define a dose that was then used in a small non-randomised study of five $\mathrm{MCl}$ subjects, used as their own controls, to define target engagement.

Main outcome measures Pharmacodynamic: $\operatorname{SAPP} \alpha$, sAPP $\beta, A \beta_{42}, \tau$ (total (t) and phosphorylated (p)) and inflammatory marker levels were time-dependently measured over $12 \mathrm{~h}$ and compared prior to and following 10 days of oral Posiphen treatment in four $\mathrm{MCl}$ subjects who completed the study. Pharmacokinetic: plasma and CSF drug and primary metabolite concentrations with estimated brain levels extrapolated from steady-state drug administration in rats.

Results Posiphen proved well tolerated and significantly lowered CSF levels of $\operatorname{SAPP} \alpha, \operatorname{sAPP} \beta, t-\tau, p-\tau$ and specific inflammatory markers, and demonstrated a trend to lower CSF $A \beta_{42}$.

Conclusions These results confirm preclinical POM studies, demonstrate that pharmacologically relevant drug/metabolite levels reach brain and support the continued clinical optimisation and evaluation of Posiphen for $\mathrm{MCl}$ and Alzheimer's disease.

\section{INTRODUCTION}

The treatment of Alzheimer's disease (AD), the most common dementing disorder of the elderly, remains an unmet medical need. ${ }^{1}$ Its hallmarks are neurodegeneration, brain atrophy and abnormal protein depositions, particularly of amyloid plaques and neurofibrillary tangles deriving from amyloid- $\beta$ peptide $(A \beta)$ and hyperphosphorylated $\tau$, respectively, ${ }^{2-4}$ resulting in progressive cognitive decline. Current approved $\mathrm{AD}$ drugs provide symptomatic relief and temporarily delay loss of cognition, but do not halt or modify disease progression. $^{5}$

The key $\mathrm{AD}$ drug target, $\mathrm{A} \beta$, is a proteolytic product of amyloid- $\beta$ precursor protein (APP) cleavage: an integral transmembrane protein concentrated at the synapse of neurons. ${ }^{4}$ APP is cleaved by $\beta$ - and $\gamma$-secretases to generate $A \beta,{ }^{2}{ }^{6}$ which assembles into oligomers that cause inflammation and target synapses to induce cellular dysfunction and impair memory. ${ }^{7-9}$ APP is additionally cleaved into a number of other bioactive $\mathrm{N}$ - and C-terminal fragments, including N-APP ${ }^{10}$ and C31 ${ }^{11} 12$ (figure 1). These fragments, likewise, may contribute to $\mathrm{AD}$ pathogenesis, making $\mathrm{APP}$ an interesting $\mathrm{AD}$ drug target to regulate. Posiphen ${ }^{\circledR}$ tartrate (Posiphen (figure 2), also known as (+)-phenserine), an APP synthesis inhibitor, ${ }^{13}$ interacts via the $5^{\prime}$-untranslated region of APP $\mathrm{mRNA}^{14}$ to inhibit ribosomal access and block APP translation. ${ }^{14-16}$ Posiphen is the chirally pure positive enantiomer of (-)-phenserine (phenserine). ${ }^{17}$ However, whereas phenserine is an acetylcholinesterase inhibitor, Posiphen lacks acetylcholinesterase activity; instead, it inhibits the translation of APP. In neuronal cultures and brain of wild type and $\mathrm{AD}$ transgenic mice, Posiphen lowered APP and $A \beta$ levels $^{13}$ is in a dose-dependent manner and hence represents an interesting candidate drug to reduce APP toxic products in humans.

We describe, herein, three phase I studies conducted under an active investigational new drug application. Initially, Posiphen's safety was assessed in healthy volunteers in a single ascending dose study and then a multiple ascending dose study (SAD and $\mathrm{MAD}$, respectively). Thereafter, using a well-tolerated dose from the former investigations, an early proof of mechanism (POM) study was conducted in patients with mild cognitive impairment (MCI), wherein time-dependent plasma and cerebrospinal fluid (CSF) samples were obtained prior to and following 10 days of Posiphen administration to permit analysis of drug-induced changes in CSF levels of secreted (s) APP $\alpha$ and APP $\beta, A \beta_{42}, \tau$ (total $(\mathrm{t})$ and phosphorylated $(\mathrm{p})$ ) and inflammatory markers. Additionally, human brain levels of Posiphen and metabolites were estimated from measured plasma and CSF samples of MCI patients in light of steady-state Posiphen plasma-brain-CSF pharmacokinetic studies performed in rats. 


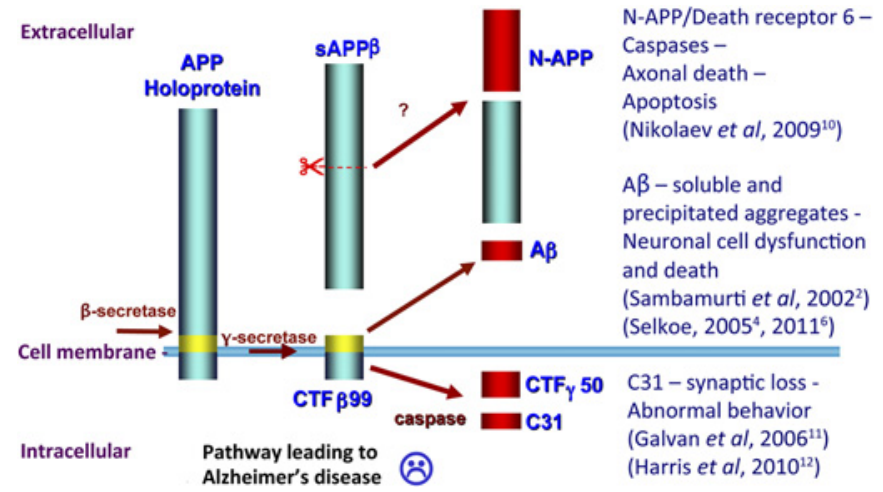

Figure 1 Schematic of amyloid- $\beta$ precursor protein (APP) processing pathway leading to $A D$. Amyloidogenic processing of APP generates $A \beta$, a hydrophobic, neurotoxic, self-aggregating 40-42 amino acid peptide that accumulates preferentially within amyloid plaques. ${ }^{4} 6$ Recent research suggests that APP processing can result in a number of toxic fragments, including $\mathrm{N}$ - and C-terminal peptides ${ }^{10} 11$ that may induce neuronal dysfunction, degeneration and death leading to the hypothesis that a number of APP fragments are toxic to nerve cells.

\section{DESIGN AND METHODS}

\section{Drug substance}

Posiphen, (3aR)-1, 3a, 8-trimethyl-1, 2, 3, 3a, 8, 8a-hexahydropyrrolo (2, 3-b) indol-5-yl phenyl-carbamate tartrate (investigational new drug \#72654) was manufactured to good manufacturing process requirements by Rhodia (Boulogne-Billancourt, France) (See online Supplemental information SI-1).

\section{Standards}

Posiphen and its metabolites, N1- and N8-norposiphen and N1, N8-bisnorposiphen, were synthesised (National Institute on Aging, Baltimore, Maryland, USA) to $>99.5 \%$ purity. ${ }^{19} 20$

\section{Animal studies}

To aid extrapolation of drug and metabolite concentrations in human brain from those measured in human plasma and CSF in the POM MCI study, Posiphen $(75 \mathrm{mg} / \mathrm{kg} /$ day continuous infusion) was administered to (male adult Fischer-44) rats under steady-state conditions and samples were simultaneously collected and drug/metabolite concentrations quantified in each compartment (See online Supplemental information SI-2).

\section{Clinical studies}

Phase I SAD

A randomised, double blind, placebo-controlled safety, tolerance and pharmacokinetic study (first in human) was performed with six groups of male and female healthy volunteers receiving serially increasing single doses of Posiphen or placebo, followed by monitoring of safety (vital signs, ECGs, clinical laboratory tests, capture of adverse events) and collection of blood and urine samples at regular intervals up to $24 \mathrm{~h}$ for preliminary pharmacokinetic analyses. The escalating doses to be studied were 10, 20, 40, 80, 160 and $240 \mathrm{mg}$ (and placebo). Due to the limiting side effects (nausea and vomiting), the highest dose studied was $160 \mathrm{mg}$ of Posiphen; the $240 \mathrm{mg}$ dose was not administered (See online Supplemental information SI-3).

The study was conducted by the PRACS Institute (East Grand Forks, Minnesota, USA) and was fully approved by their Institutional Review Board.

\section{Phase I MAD}

A randomised, double blind, placebo-controlled safety, tolerance, pharmacokinetic study was performed with six of eight male and six of eight female healthy volunteers in each of three successive groups being administered one of three serially increasing, multiple dose regimens of Posiphen and two male and two female subjects in each treatment group receiving placebo. Safety (vital signs, ECGs, clinical laboratory tests, capture of adverse events) was monitored throughout the study and blood and urine samples were collected. The escalating regimens were 20,40 and $60 \mathrm{mg}$, and placebo four times a day for 7 or 10 days. Plasma obtained from blood samples was analysed as described above (See online Supplemental information SI-4).

For inclusion/exclusion criteria see online Supplemental information SI-5.

The study was likewise conducted by the PRACS Institute and was fully approved by their Institutional Review Board.

Phase I early POM with pharmacokinetics and pharmacodynamics (ClinicalTrials.gov Identifier: NCT01072812)

An open-label study was performed in which five healthy male and female MCI patients (three male and two female patients) received Posiphen at $4 \times 60 \mathrm{mg} /$ day (total $240 \mathrm{mg} /$ day) for 10 days. This dosing regimen was well tolerated in the earlier MAD study. To avoid potential inter-subject variability, subjects were used as their own controls. Specifically, serial plasma and
Figure 2 Chemical structures of Posiphen and primary metabolites, and their plasma, brain and CSF concentrations in $(A)$ rats and $(B)$ mild cognitive impairment (MCI) subjects following Posiphen administration. (A) Simultaneous plasma $(\mathrm{ng} / \mathrm{ml})$, brain (ng/g wet weight) and CSF (ng/ml) levels of Posiphen and metabolites measured under steady-state conditions following Posiphen $(75 \mathrm{mg} / \mathrm{kg} / \mathrm{day} \times 10$ days by continuous administration subcutaneous pump). (B) In an equivalent $\mathrm{MCl}$ phase I study following 10 days of Posiphen $(4 \times 60 \mathrm{mg} /$ day), plasma $(\mathrm{ng} / \mathrm{ml})$ and CSF $(\mathrm{ng} / \mathrm{ml})$ levels of Posiphen and metabolites were measured, and extrapolated brain levels were determined from steady-state animal data.
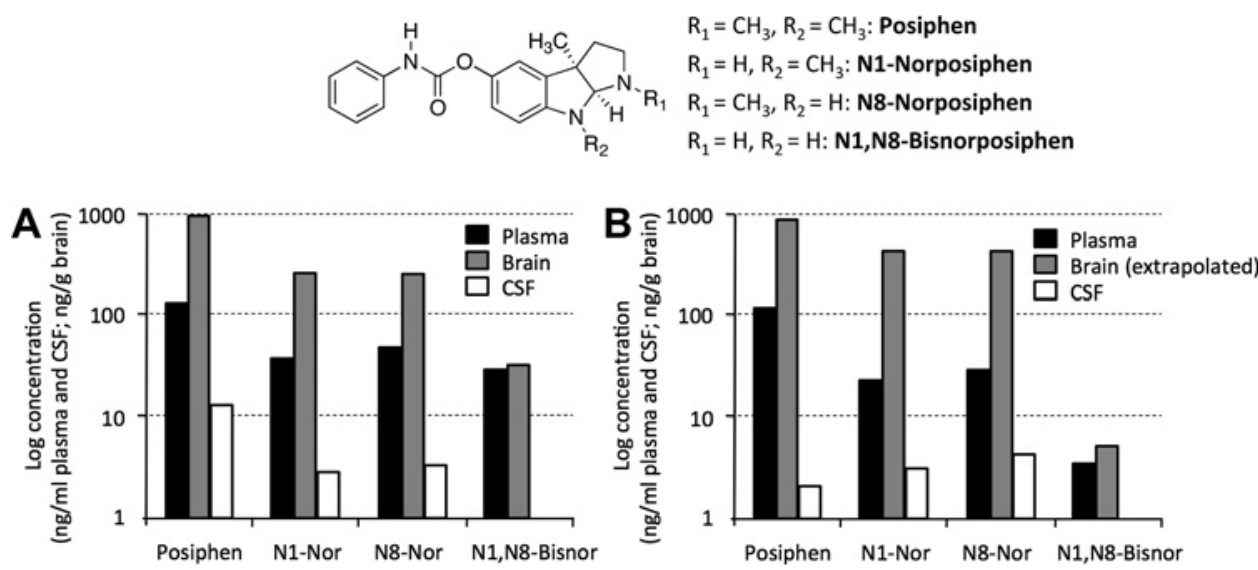
lumbar CSF samples were collected via an indwelling catheter over $12 \mathrm{~h}$ (at $0,1,1.5,2,3,4,6,8$ and $12 \mathrm{~h}$ ) initiated at the same time of day 1 day prior to the start of dosing to obtain timedependent baseline control data, and then at the exact same times immediately after the last dose was administered. ${ }^{21} 22$ This paradigm was chosen to control for potential circadian alterations in pharmacodynamics markers within each subject. Samples were frozen and then stored at $-80^{\circ} \mathrm{C}$. CSF and plasma samples were matched and were analysed for (i) pharmacokinetics of Posiphen and metabolites (N1-norposiphen, N8norposiphen and N1, N8-bisnorposiphen) (figure 2) and (ii) pharmacodynamic studies involving measurement of the following proteins: $\mathrm{sAPP} \alpha, \mathrm{sAPP} \beta, A \beta_{42}, t-\tau, \mathrm{p}-\tau$, complement 3 , factor $\mathrm{H}$, monocyte chemotactic protein-1 (MCP-1), the inflammation marker and chitinase-like protein YKL-40, and soluble cluster of differentiation 14 (sCD14). One MCI subject withdrew from the POM study on day 1 (table 1 legend); hence analyses were undertaken on four subjects in relation to pharmacokinetic and pharmacodynamics measures.

Subjects were male or postmenopausal females, between 55 and 80 years of age, with self-reported memory complaints that were corroborated by spouse or companion or caregiver as appropriate, and memory difficulties as measured on neuropsychological tests. MCI was determined according to Petersen's criteria $^{23}$ with a Mini Mental Status Examination score $\geq 24$, cut-off score on the logical memory II delayed paragraph recall subtest of the Wechsler Memory Scale Revised, Clinical Dementia Rating of 0.5 with a memory box score of 0.5 or 1.0 . Inclusion/ exclusion criteria were in accordance with those described in online Supplemental information SI-6.

To provide comparative healthy control data, a CSF sample was similarly acquired by spinal tap at 08:00 AM from four healthy volunteers who were not treated with Posiphen. This CSF was analysed for the same key pharmacodynamic factors as those described for the MCI patients prior and following Posiphen treatment.

The study was conducted by the CEDRA/World Wide Clinical Trials (King of Prussia, Pennsylvania, USA) and was fully approved by their Institutional Review Board.

\section{Biochemical pharmacodynamic assays}

Four independent laboratories and multiple assay methods were used to quantify the CSF biomarkers analysed in this study.

Meso Scale Discovery (MSD) assays were used for quantification of sAPP $\alpha$ and SAPP $\beta$ (human Alzheimer's panel three sAPP $\alpha /$ sAPP $\beta$ assay kit (catalogue\#: K15120E-1, MSD, Gaithersburg, Maryland, USA)) from $25 \mu$ of CSF. The plates were measured by multiplex analyser (SI2400A) and data evaluated by MSD discovery workbench data analysis toolbox software. The same platform was used to measure MCP-1 (also called CCL2) concentrations in CSF, as previously described. ${ }^{24}$

AlphaLISA kits from Perkin Elmer were used to quantify sAPP $\alpha$ (catalogue\#: AL254C, Perkin Elmer, Waltham, Massachusetts, USA), sAPP $\beta$ (catalogue\#: AL232C), A $\beta_{42}$ (catalogue\#: $\mathrm{AL} 276 \mathrm{C}$ ), $\mathrm{A} \beta_{40}$ (catalogue\#: AL275C) and $\mathrm{t}-\tau$ (catalogue\#: AL271C) from $5 \mu \mathrm{l}$ of an undiluted CSF sample which was added to an AlphaPlate-384 (catalogue\#: 6005350).

Innogenetics kits were used to measure CSF $t-\tau$ and $p-\tau$ levels by INNO-BIA AlzBio3 kit (Innogenetics, Gent, Belgium) with CSF samples diluted 1:4 in diluent. Samples were analysed using a LiquiChip Luminex 200 Workstation (Oiagen, Valencia, California, USA).

The proteins $\mathrm{C} 3$ and $\mathrm{FH}$ in CSF were measured with a kit made by Millipore Corporation (Billerica, Massachusetts, USA), following the manufacturer's instructions with procedures similar to those described for synuclein assay. ${ }^{25}$

CSF levels of YKL-40 and sCD14 were analysed with commercial ELISAs (R\&D Systems, Minneapolis, Minnesota, USA). The CSF was diluted 100 times for the YKL-40 and sCD14 analyses.

\section{Posiphen and metabolite pharmacokinetic assays}

Concentrations of Posiphen, N1-norposiphen, N8-norposiphen and N1, N8-bisnorposiphen in human plasma and CSF as well as rat plasma, brain and CSF samples were determined by LC-MS/MS at Absorption Systems (Exton, Pennsylvania, USA) (See online Supplemental information SI-7). Calibration ranges for each analyte ranged from $1000 \mathrm{ng} / \mathrm{ml}$ to $1 \mathrm{ng} / \mathrm{ml}$ (or $\mathrm{ng} / \mathrm{g}$ for brain) in plasma, brain and CSF matrices. The detection limit was $0.025 \mathrm{ng} / \mathrm{ml}$.

\section{Statistical analysis}

All assay data collected were analysed using a repeated measures mixed model analysis of variance. The model included Day (Day 11/Day 0) as a fixed effect, Time (nine time points per person between 0 and $12 \mathrm{~h}$ ) as a repeated measure effect and Patient (18 samples per person in total) as a random effect. Compound symmetry was assumed as an appropriate covariance pattern between observations on the same patient, which provided a reasonable model fit. Assumptions of constant variance, normality or residuals and parallelism were used to assess acceptability of the statistical model. Data are presented as means \pm SEs, unless otherwise stated. The statistical evaluations were undertaken by Data Magik (Salisbury, UK).

\section{RESULTS}

\section{Rat pharmacokinetics of Posiphen/metabolites}

The comparative plasma, brain and CSF levels of Posiphen and three primary metabolites in rats following steady-state Posiphen infusion are shown in table 2 and figure 2A. Posiphen was the primary compound in each compartment, with the N1- and N8-metabolites reaching $39.1 \%$ and $25.8 \%$ of Posiphen levels in plasma, respectively, and N1, N8-bisnorposiphen 20.5\%. In accordance with their high lipophilicity (ClogP value, table 2), substantial brain entry of Posiphen and metabolites was evident whereas aqueous CSF levels were low. Specifically, steady-state brain concentrations were greater than concomitant plasma levels, providing high brain to plasma ratios (Posiphen: 6.8, N1-norposiphen: 3.8, N8-norposiphen: 5.8 and N1, N8-bisnorposiphen: 1.3) with CSF levels reaching only approximately $1 \%$ of brain levels.

\section{SAD study (healthy volunteers)}

Safety

Posiphen was well tolerated by healthy male and female volunteers at single doses from 10 to $80 \mathrm{mg}$. A $160 \mathrm{mg}$ dose was associated with an increased incidence of nausea and vomiting (four subjects were nauseous and three vomited). Adverse events were either mild or moderate; none were severe. No higher doses were administered. Posiphen $80 \mathrm{mg}$ was determined as the no observed adverse effect level (table 1).

\section{Pharmacokinetics}

Posiphen, at all doses, was absorbed rapidly (mean Tmax: $1.3-1.6 \mathrm{~h}$ ) and cleared from the circulation biphasically (terminal half life: $3.7-4.3 \mathrm{~h}$ ), independent of dose. Posiphen 
Table 1 Summary of adverse events (AEs). Treatment-related AEs that occurred in more than one subject in the Posiphen or placebo groups or in the entire cohort are summarised by dose. In all three (AX-PO-101, AX-P0-102, QR-12001) studies, males and female subjects were combined, as there was no apparent difference between the sexes regarding their tolerance to Posiphen. Of note, markers of hepatic and renal function were additionally analysed and were unaltered by drug

\begin{tabular}{|c|c|c|c|c|c|c|}
\hline \multicolumn{7}{|c|}{ AEs in healthy male and female volunteers } \\
\hline \multicolumn{7}{|c|}{ Single ascending dose $(n=72)$} \\
\hline$\overline{\mathrm{AE}}$ & $10 \mathrm{mg}(\mathrm{n}=10)$ & $20 \mathrm{mg}(\mathrm{n}=20)$ & $40 \mathrm{mg}(\mathrm{n}=10)$ & $80 \mathrm{mg}(\mathrm{n}=10)$ & $160 \mathrm{mg}(\mathrm{n}=10)$ & Placebo $(n=12)$ \\
\hline All AEs, moderate & $0(0)$ & $2(10.0)$ & $0(0)$ & $0(0)$ & $4(40.0)$ & $1(8.3)$ \\
\hline All AEs, severe & $0(0)$ & $1(5.0)$ & $0(0)$ & $0(0)$ & $0(0)$ & $1(8.3)$ \\
\hline \multicolumn{7}{|l|}{ Gastrointestinal symptoms } \\
\hline Nausea & $0(0)$ & $2(10.0)$ & $0(0)$ & $0(0)$ & $4(40.0)$ & $0(0)$ \\
\hline Vomiting & $0(0)$ & $0(0)$ & $0(0)$ & $0(0)$ & $3(30.0)$ & $0(0)$ \\
\hline \multicolumn{7}{|l|}{ Nervous system symptoms } \\
\hline Dizziness & $0(0)$ & $3(15.0)$ & $1(10.0)$ & $3(30.0)$ & $4(40.0)$ & $2(16.7)$ \\
\hline Fainting & $0(0)$ & $1(5.0)$ & $0(0)$ & $0(0)$ & $0(0)$ & $1(8.3)$ \\
\hline \multicolumn{7}{|l|}{ General symptoms } \\
\hline Feeling hot & $0(0)$ & $2(10.0)$ & $0(0)$ & $0(0)$ & $0(0)$ & $0(0)$ \\
\hline Heart rate increased & $2(20.0)$ & $1(5.0)$ & $0(0)$ & $0(0)$ & $0(0)$ & $2(16.7)$ \\
\hline Orthostatic hypotension & $0(0)$ & $1(5.0)$ & $0(0)$ & $0(0)$ & $0(0)$ & $1(8.3)$ \\
\hline \multicolumn{7}{|c|}{ AEs in healthy male and female volunteers } \\
\hline \multicolumn{7}{|c|}{ Multiple ascending dose $(n=48)$} \\
\hline$\overline{\mathrm{AE}}$ & \multicolumn{2}{|l|}{$4 \times 20 \mathrm{mg}(\mathrm{n}=12)$} & $4 \times 40 \mathrm{mg}(\mathrm{n}=12)$ & \multicolumn{2}{|c|}{$4 \times 60 \mathrm{mg}(\mathrm{n}=12)$} & Placebo $(n=12)$ \\
\hline All AEs, moderate & \multicolumn{2}{|l|}{$2(16.7)$} & $0(0)$ & \multicolumn{2}{|l|}{$1(8.3)$} & $2(16.7)$ \\
\hline All AEs, severe & \multicolumn{2}{|l|}{$0(0)$} & $0(0)$ & \multicolumn{2}{|l|}{$0(0)$} & $0(0)$ \\
\hline \multicolumn{7}{|l|}{ Gastrointestinal symptoms } \\
\hline Nausea & \multicolumn{2}{|l|}{$1(8.3)$} & $0(0)$ & \multicolumn{2}{|c|}{$2(16.7)$} & $1(8.3)$ \\
\hline Vomiting & \multicolumn{2}{|l|}{$0(0)$} & $0(0)$ & \multicolumn{2}{|c|}{$2(16.7)$} & $0(0)$ \\
\hline \multicolumn{7}{|l|}{ Nervous system symptoms } \\
\hline Dizziness & \multicolumn{2}{|l|}{$2(16.7)$} & $2(16.7)$ & \multicolumn{2}{|c|}{$3(25.0)$} & $1(8.3)$ \\
\hline \multicolumn{7}{|l|}{ General symptoms } \\
\hline Abdominal pain & \multicolumn{2}{|l|}{$1(8.3)$} & $0(0)$ & \multicolumn{2}{|l|}{$0(0)$} & $0(0)$ \\
\hline Headache & $2(16.7)$ & & $3(25.0)$ & $1(8.3)$ & & $2(16.7)$ \\
\hline Other & $5(41.2)$ & & $1(8.3)$ & $1(8.3)$ & & $6(50.0)$ \\
\hline AEs in $\mathrm{MCl}$ patients & & & & & & \\
\hline Multiple dose $(n=5)$ & & & & & & \\
\hline Adverse event & & & & & & $4 \times 60 \mathrm{mg}(n=5)$ \\
\hline All AEs, moderate & & & & & & $0(0) 1^{*}$ \\
\hline All AEs, severe & & & & & & $0(0)$ \\
\hline Gastrointestinal symptoms & & & & & & \\
\hline Nausea & & & & & & $1(20) 1^{*}$ \\
\hline Vomiting & & & & & & $0(0) 1^{*}$ \\
\hline Nervous system symptoms & & & & & & \\
\hline Dizziness & & & & & & $1(20.0)$ \\
\hline General disorders & & & & & & \\
\hline General pain & & & & & & $1(20.0)$ \\
\hline Headache & & & & & & $5(100.0)$ \\
\hline Other & & & & & & $5(100.0)$ \\
\hline
\end{tabular}

The AEs, dizziness/fainting and orthostatic hypotension, were the most frequently observed safety observations during the course of this study. Orthostatic hypotension was observed in Posiphen treated people, but occurred with comparable frequency in placebo subjects. Some subjects experienced orthostatic effects at multiple measurement points postdose, but there was no apparent correlation between time of occurrence and plasma concentration of Posiphen. Dizziness, nausea and vomiting increased with Posiphen doses and at 160 mg there was a statistically significant effect of Posiphen on these two measures. In the multiple ascending dose studies there was a trend, but no statistically significant effect, in the $4 \times 60 \mathrm{mg} /$ day group.

${ }^{*}$ One person had leg cramps and was nauseous during the catheterisation (predrug). This subject vomited and dropped out after the second and before the third 60 mg dose on day 1. $\mathrm{MCl}$, mild cognitive impairment.

systemic availability increased more than linearly with increasing dose, resulting in a disproportionately large increase in Cmax and

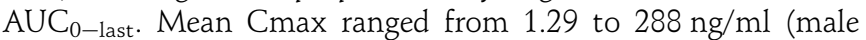
subjects) and 6.22 to $480 \mathrm{ng} / \mathrm{ml}$ (female subjects) as the dose increased from 10 to $160 \mathrm{mg}$. Comparable increases were determined for mean $\mathrm{AUC}_{0-\text { last }}, 1.63-998$ and $12.1-1530 \mathrm{ng} . \mathrm{h} / \mathrm{ml}$, respectively, over the same dose range (data not shown).

\section{MAD study (healthy volunteers)}

Safety

Posiphen doses up to $4 \times 60 \mathrm{mg}$ daily $\times 10$ days were well tolerated. This $4 \times 60 \mathrm{mg}$ dose produced a small but statistically insignificant difference from placebo regarding gastrointestinal side effects and dizziness, and hence $4 \times 60 \mathrm{mg}$ four times a day was determined the no observed adverse effect level (table 1). 
Table 2 Mean pharmacokinetic parameters for Posiphen and primary metabolites in rat and $\mathrm{MCl}$ patients

\begin{tabular}{|c|c|c|c|c|c|c|}
\hline $\begin{array}{l}\text { Posiphen and metabolites } \\
\text { Analyte }\end{array}$ & Parameters & $\begin{array}{l}\text { Human plasma } \\
(\mathrm{ng} / \mathrm{ml}) \pm \mathrm{SD}\end{array}$ & $\begin{array}{l}\text { Human CSF } \\
(\mathrm{ng} / \mathrm{ml}) \pm \mathrm{SD}\end{array}$ & $\begin{array}{l}\text { Rat plasma } \\
(\mathrm{ng} / \mathrm{ml}) \pm \mathrm{SD}\end{array}$ & $\begin{array}{l}\text { Rat brain } \\
(\mathrm{ng} / \mathrm{g}) \pm \text { SD }\end{array}$ & $\begin{array}{l}\text { Rat CSF } \\
(\mathrm{ng} / \mathrm{ml}) \pm \mathrm{SD}\end{array}$ \\
\hline Posiphen & Cmax & $118.5 \pm 24.8$ & $1.6 \pm 0.6$ & $144 \pm 69.5$ & $979 \pm 429$ & $9.5 \pm 4.6$ \\
\hline${ }^{*} \mathrm{C} \log \mathrm{P}=2.22$ & $\mathrm{AUC}_{0-\text { last }}$ & $570 \pm 235.4$ & & & & \\
\hline N1-Norposiphen & Cmax & $25.6 \pm 6.7$ & $1.7 \pm 0.7$ & $56.3 \pm 8.7$ & $213 \pm 27.9$ & $2.8 \pm 1.1$ \\
\hline $\operatorname{Clog} P=1.25$ & $\mathrm{AUC}_{0-\text { last }}$ & $214.4 \pm 77.1$ & & & & \\
\hline N8-Norposiphen & Cmax & $31 \pm 7.1$ & $3.2 \pm 1.2$ & $37.2 \pm 9.1$ & $216 \pm 28.6$ & $2.8 \pm 5.8$ \\
\hline$C \log P=1.00$ & $A U C_{0-\text { last }}$ & $261.3 \pm 91.3$ & & & & \\
\hline N1, N8-bisnorposiphen & Cmax & $3.8 \pm 1.2$ & Not detected & $29.5 \pm 10.8$ & $37.2 \pm 10.5$ & Not detected \\
\hline$C \log P=0.53$ & $\mathrm{AUC}_{0-\text { last }}$ & $36.9 \pm 12.5$ & & & & \\
\hline
\end{tabular}

Rat: A comparison is shown of plasma, brain and CSF levels of Posiphen and metabolites undertaken under steady-state conditions, which was achieved by continuous administration of Posiphen $75 \mathrm{mg} / \mathrm{kg} /$ day for 10 days by osmotic mini pump. In rat plasma (ng/ml) and brain (ng/g wet weight), Posiphen proved to be the primary drug compound, with the two major metabolites, N1- and N8-norposiphen, comprising up to $39.1 \%$ of Posiphen in plasma and up to $22 \%$ of Posiphen in brain at the Cmax. The third metabolite, N1, N8-bisnorposiphen, reached $20.4 \%$ of Posiphen in plasma and 3.8\% of Posiphen at Cmax in brain. Human: the pharmacokinetic parameters of Posiphen and metabolites are shown in plasma and CSF of MCl patients after 10 days of $4 \times 60 \mathrm{mg} /$ day repeat dose oral administration, male and female subjects combined. As expected from the rodent data, the two primary metabolites N1- and N8-norposiphen constitute approximately $20 \%$ of Posiphen at the Cmax, with the third metabolite N1, N8-bisnorposiphen being a minor component and reaching only $3 \%$ of Posiphen Cmax. The timedependent pharmacokinetic profiles of Posiphen and metabolites are provided in figure 3.

*The ClogP value is an established measure of a compound's lipid versus water solubility, with a positive value associated with a preference for the lipid phase.

$\mathrm{MCl}$, mild cognitive impairment.

\section{Pharmacokinetics}

Posiphen, at all doses, was absorbed rapidly ( $\max =1.2$ to $1.7 \mathrm{~h}$ ) and cleared from the circulation biphasically (terminal half life of $4.3-4.7 \mathrm{~h}$ ). As with the SAD study, the systemic availability of Posiphen increased more than linearly with increasing dose, resulting in disproportionately large increases in Cmax and AUC values.

\section{POM study (MCI patients)}

Safety

Posiphen $(4 \times 60 \mathrm{mg}$ daily $\times 10$ days $)$ in MCI subjects showed a similar safety profile as found in the healthy volunteers (table 1).

\section{Pharmacokinetics}

Mean Cmax plasma concentrations of Posiphen and metabolites are shown in Table 2 and figure $2 \mathrm{~B}$, and the time-dependent profiles are provided in figure 3 . Similar to rat, Posiphen was the primary drug species, with the N1- and N8-metabolites initially accounting for $21.6 \%$ and $26.2 \%$ of Posiphen's Cmax. Total timedependent levels $\left(\mathrm{AUC}_{0-\text { last }}\right.$, table 2 ) were approximately $50 \%$ of Posiphen. Contrasting with rat studies, N1, N8-bisnorposiphen represented a minor metabolite in humans, accounting for $3.2 \%$ of Cmax and 6.5\% total time-dependent Posiphen levels.
Calculated pharmacokinetic parameters for Posiphen in plasma of MCI patients were similar to those in healthy volunteers (SAD and MAD). Posiphen mean Tmax was 1.3-1.6 h, mean terminal half life $4.0-5.5 \mathrm{~h}$, apparent volume of distribution: $2171 \pm 339 \mathrm{l}$ and total body clearance $310 \pm 72 \mathrm{l} / \mathrm{h}$. The four times a day regimen resulted in some accumulation of Posiphen in plasma and accumulation of Posiphen/metabolites in CSF (figure 3)

Illustrated in figure $2 \mathrm{~B}$ are Posiphen and metabolite plasma, CSF and estimated brain levels for MCI patients following 10 day Posiphen dosing. Extrapolated brain levels for MCI patients were determined by applying the rat plasma/CSF/brain data (figure 2A) to the human plasma and CSF data (figure 2B). This estimate predicts Posiphen brain levels approaching $1 \mathrm{ug} / \mathrm{g}$ or approximately $3.5 \mu \mathrm{M}$ for a dose of $4 \times 60 \mathrm{mg} /$ day.

\section{Pharmacodynamics}

Drug-induced differences in CSF biomarkers for MCI patients are shown in table $3 \mathrm{~A}$, determined by comparing the predrug and postdrug biomarker levels at each time point within the same subject to control for both circadian changes and intersubject variability. The majority of the biomarkers were analysed by two different techniques within two independent institutions to cross-validate the data. In all cases, the direction
Figure 3 Time-dependent (A) plasma and (B) CSF Posiphen and metabolite levels following the final dose of 10 day Posiphen administration $(4 \times 60 \mathrm{mg})$. Semi-log plot of serial time-dependent Posiphen and metabolite (N1- and N8-norposiphen and N1, N8bisnorposiphen) concentrations in plasma and CSF obtained following the final dose of a 10 day Posiphen $(4 \times 60 \mathrm{mg})$ schedule $(\mathrm{n}=4 \mathrm{MCl}$ subjects, mean $\pm \mathrm{SD}$ ).
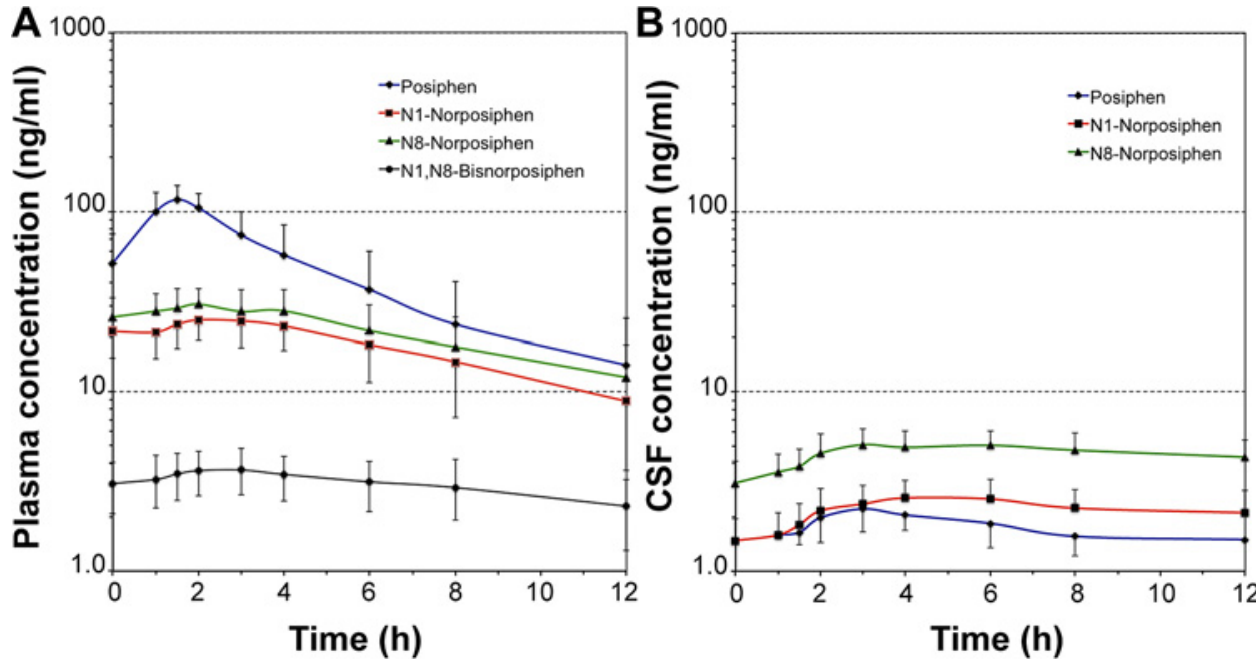
Table 3 (A) AD biomarkers and (B) inflammatory biomarkers in CSF of $\mathrm{MCl}$ subjects after 10 days of Posiphen treatment

\begin{tabular}{|c|c|c|c|c|c|}
\hline Human biomarker & CSF $\%$ of time 0 & SE & p Value & Assay & Laboratory \\
\hline \multicolumn{6}{|l|}{ (A) AD biomarkers } \\
\hline \multirow[t]{2}{*}{$\operatorname{sAPP} \alpha$} & $-59.9 \%$ & 0.231 & 0.0006 & AlphaLisa & V. John/Buck Institute \\
\hline & $-34.1 \%$ & 0.659 & 0.0661 & MSD & MY Chan/QR Pharma \\
\hline \multirow[t]{2}{*}{ sAPP $\beta$} & $-57.7 \%$ & 0.361 & 0.0001 & AlphaLisa & V. John/Buck Institute \\
\hline & $-34.0 \%$ & 1.516 & 0.0901 & MSD & MY Chan/OR Pharma \\
\hline \multirow[t]{2}{*}{$A \beta 42$} & $-45.2 \%$ & 1.726 & 0.0995 & AlphaLisa & V. John/Buck Institute \\
\hline & $-51.4 \%$ & 1.119 & 0.0533 & Innogenetics & C. Pan/Inarian \\
\hline \multirow[t]{2}{*}{$\tau$ (total) } & $-46.2 \%$ & 0.538 & 0.0020 & AlphaLisa & V. John/Buck Institute \\
\hline & $-74.1 \%$ & 0.259 & 0.0150 & Innogenetics & C. Pan/Inarian \\
\hline$\tau$ (Phosphorylated) & $-61.0 \%$ & 0.195 & 0.0005 & Innogenetics & C. Pan/Inarian \\
\hline \multicolumn{6}{|c|}{ (B) Inflammatory biomarkers } \\
\hline Complement C3 & $-86.9 \%$ & 0.139 & 0.0007 & Millipore & C. Pan/Inarian \\
\hline Factor FH & $23.7 \%$ & 1.237 & 0.4988 & Millipore & C. Pan/Inarian \\
\hline MCP-1 & $-87.5 \%$ & 4.813 & 0.0007 & MSD & H. Zetterberg/Univ. Göteborg \\
\hline YKL-40 & $-72.7 \%$ & 2.2 & 0.0113 & R\&D Systems & H. Zetterberg/Univ. Göteborg \\
\hline sCD14 & $-26.1 \%$ & 1.7 & 0.1159 & R\&D Systems & H. Zetterberg/Univ. Göteborg \\
\hline
\end{tabular}

of change was the same. Specifically, Posiphen lowered SAPP $\alpha$ and sAPP $\beta$ levels by $-59.9 \%$ and $-57.7 \%$, respectively, assessed by the AlpaLisa assay, and by $-34.1 \%$ and $-34 \%$, respectively, assessed by the MSD assay, in accordance with Posiphen's proposed mechanism of action to inhibit APP expression. ${ }^{13} 1418$ In line with this, $A \beta_{42}$ demonstrated a trend to reduction $(-45.2 \%$, as assessed by the AlphaLisa assay and $-51.4 \%$ as assessed by the Innogenetics assay). In addition, Posiphen significantly reduced levels of $\mathrm{t}-\tau(-74.1 \%$, as assessed by the Innogenetics assay and $-46.2 \%$, as assessed by the AlphaLisa assay) and $p-\tau$ ( $-61 \%$, as assessed by the Innogenetics assay).

Posiphen's actions on CSF inflammation markers are shown in table 3B. A significant lowering of pro-inflammatory, C3 $(-86.9 \%)$ and microglial activation markers, MCP-1 (-87.5\%) and YKL-40 (-72.7\%), was evident. By contrast, sCD14, associated with early innate immune response to bacterial and viral infection, ${ }^{26}$ and the complement control protein, factor $\mathrm{H}$, were unaffected by Posiphen ( $-26.1 \%$ and $+23.7 \%$, respectively).

Analysis of CSF samples obtained from four healthy Posiphen naive volunteers under conditions similar to those for the $\mathrm{MCI}$ subjects permitted comparison between untreated and treated MCI patients and healthy untreated volunteers. Figure 4 demonstrates that Posiphen administration to MCI patients lowers $\operatorname{sAPP} \alpha, \operatorname{sAPP} \beta$ and $t-\tau$ to levels present in healthy volunteers.

\section{DISCUSSION}

Posiphen was shown in 72 healthy volunteers to be safe in SAD and 53 subjects in MAD and POM studies of 7-10 day administration up to levels determined as greater than fivefold the effective dose. This effective dose was determined both by comparing the extrapolated molar concentration in brain with the $50 \%$ effective concentration to inhibit APP in neuronal cultures, as well as from animal studies. ${ }^{13}$ The human pharmacokinetics of Posiphen/metabolites and extrapolation of data determined from rodents suggests that Posiphen readily enters the brain and achieves levels 6.8-fold higher than in plasma at steady-state, in accordance with its high lipophilicity (ClogP value 2.2$)$. Its hydrophobicity and protein binding capacity $(96 \%$ of Posiphen/ metabolites bind to brain proteins) likely limit levels of Posiphen/metabolites found in CSF of both rodents and MCI patients. Interestingly, Posiphen half life in CSF of $\mathrm{MCI}$ patients proved to be longer than its half life in plasma, $>12 \mathrm{~h}$ versus approximately $5 \mathrm{~h}$, respectively (figure 3 ). Consistent with its longer half life within the central nervous system, Posiphen's APP, $\tau$ and inflammation lowering activity lasted longer than the recorded $12 \mathrm{~h}$ of sampling. Recent studies in neuronal cultures indicate that Posiphen's APP lowering actions extend for numerous hours following wash-off and, additionally, are likewise maintained within the brain of transgenic $A D$ mice for over $9 \mathrm{~h}$ after cessation of dosing (Sambamurti $\mathrm{K}$, Medical University of $\mathrm{S}$. Carolina, personal communication). The extended duration of Posiphen/metabolites in CSF/brain together with the prolonged inhibition of APP and $\tau$ expression may permit once a day dosing and is a focus of current studies.

Our early POM study in MCI patients focused on evaluating target engagement demonstrates that Posiphen lowers both sAPP $\alpha$ and SAPP $\beta$ in CSF, consistent with its preclinical actions $^{13} 18 \quad 27$ and ability to inhibit the translation of APP mRNA via an iron response element within its $5^{\prime}$-untranslated region. ${ }^{14} 1628$ The trend of a Posiphen-induced reduction in CSF $\mathrm{A} \beta_{42}$ in $\mathrm{MCI}$ subjects is likewise in line with its known action to inhibit APP synthesis, as $A \beta_{42}$ is a downstream product and is in accordance with the described decline in CSF SAPP $\beta$ in $\mathrm{MCI}$ subjects as well as of $A \beta_{42}$ levels in preclinical studies. ${ }^{13-15} 182728$ A separate more limited analysis of $A \beta_{40}$ (AlphaLisa) in CSF collected at 3 and $8 \mathrm{~h}$ prior to and following Posiphen administration in MCI subjects provided reduction trends of $-32 \%$ and $-37 \%$, respectively. A caveat of early CNS target engagement investigational studies is the small patient number required to, on one hand, adequately demonstrate pharmacologically driven biological activity in the brain as a result of drug interaction with its intended target to provide proof-of-concept and, on the other hand, protect patients from exposure to potentially inactive or toxic drugs. ${ }^{29}$ In our Posiphen study, this was undertaken on five MCI subjects, one of whom withdrew, allowing biomarker analyses on four. However, as patients were used as their own controls at 0 day (prior to Posiphen treatment) and as 
Figure 4 Comparison of $\operatorname{sAPP} \alpha$, sAPP $\beta$ and $t-\tau$ between untreated and treated mild cognitive impairment (MCl) patients and healthy volunteers. Posiphen $(4 \times 60 \mathrm{mg} /$ day $\times 10$ days $)$ administered to $\mathrm{MCl}$ patients for 10 days lowers their CSF levels of $\operatorname{sAPP} \alpha, \operatorname{sAPP} \beta$ and $t-\tau$ to those determined in healthy (Posiphen naive) volunteers. Biomarker concentrations are expressed as a per cent of $\mathrm{MCl}$ predrug values (assigned as 100\%) and combined all data across assays (AlphaLisa and MSD for sAPP $\alpha$ and SAPP $\beta$, and AlphaLisa and Innogenetics for $t-\tau$ ) in Table 3. Whereas there were significant Posiphen-induced declines in $\mathrm{MCl}$ subjects (see Table 3 for statistical analyses), no dramatic differences were evident between Posiphen-treated $\mathrm{MCl}$ subjects and healthy volunteers.
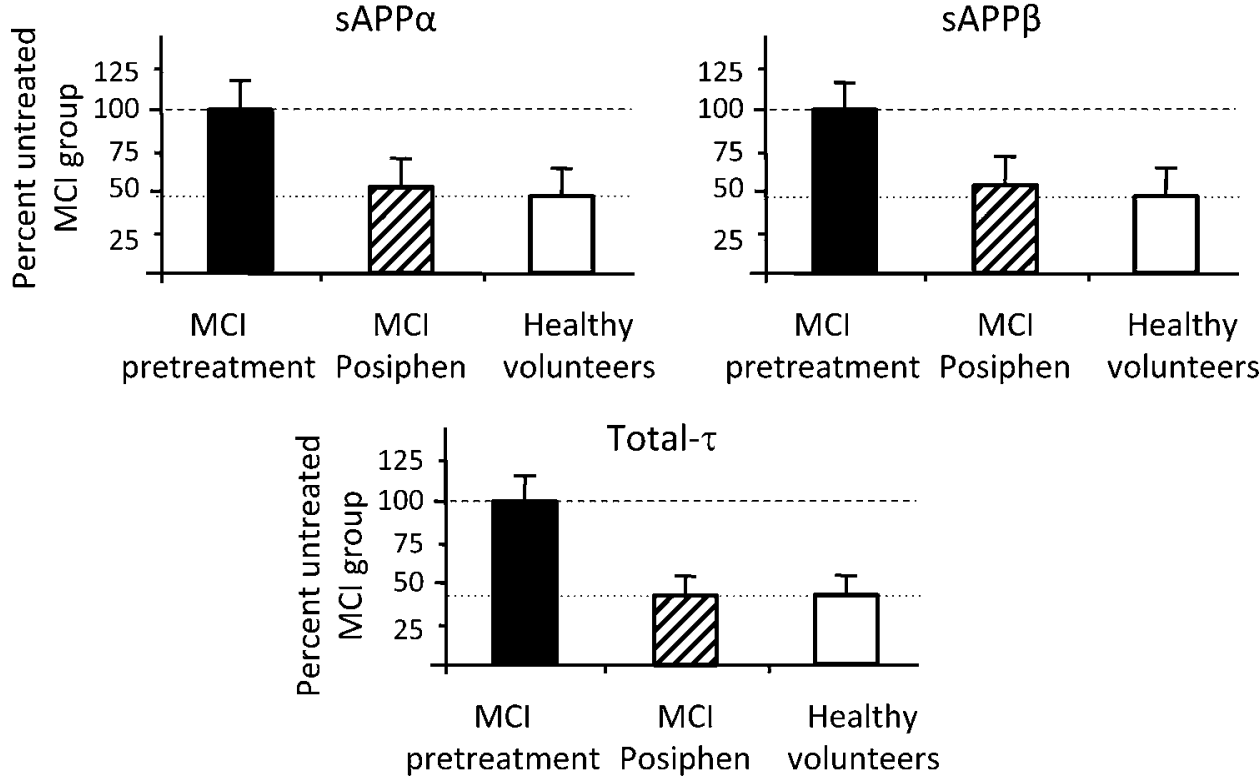

test subjects following 10 days Posiphen treatment, the POM study design limited the potential effect of often large intersubject variability ${ }^{30}{ }^{31}$ thereby permitting statistical analyses on data derived from this small patient number. In this regard, individual patient data analyses are shown in figure 5. Clearly evident is the inter-subject difference in biomarker levels under naive (day 0 ) conditions (determined as the mean value \pm SD of the nine timed samples across the $12 \mathrm{~h}$ sampling period). Evident also is the sometimes high variance around the mean biomarker value for each individual related to the time-dependent change (consistent with the circadian pattern reported by others ${ }^{31}$ ) in biomarker levels over the $12 \mathrm{~h}$ study. Consistently across all individuals within figure 5, 10 day Posiphen administration lowered mean levels of sAPP $\alpha$, sAPP $\beta, A \beta_{42}, t-\tau, p-\tau$ and $C 3$, but not factor $\mathrm{H}$. Importantly, the time-dependent analysis of biomarker levels within the same individual, by matching exact same times predrug versus postdrug (table 3A, B), allowed determination of Posiphen-induced differences in such a small patient number $(\mathrm{N}=4)$ in the presence of large inter-subject and time-dependent biomarker differences. Of significance, the
Figure 5 Difference in biomarkers between day 0 (naive) and 10 day Posiphen ( $4 \times 60 \mathrm{mg} /$ day) administration. As the effect of Posiphen on the analysed biomarkers remained present over $12 \mathrm{~h}$, we were able to calculate the mean of the nine samples (collected $0-12 \mathrm{~h}$ ) on day 0 (naive) and compare this with the mean of the nine samples (collected over the same $0-12 \mathrm{~h}$ ) after 10 day Posiphen administration. Each bar hence represents the mean of repeat measures over $12 \mathrm{~h}$ with $\mathrm{SD}$ for each of the four patients $(A, B, C, D)$ who completed the study. Whereas the absolute biomarker level differs between subjects, for each individual subject the post Posiphen values are consistently lower than the predrug (naive) values for all shown markers with the exception of factor $\mathrm{H}$, consistent with its regulatory and not pro-inflammatory role. For this figure, data are derived from the AlphaLisa for $\operatorname{sAPP} \alpha$ and SAPP $\beta$, Innogenetics for $A \beta_{42}, p-\tau$ and $t-\tau$, and Millipore for $C 3$ and factor $\mathrm{H}$. In general, assay values are in agreement with literature values. ${ }^{38} \mathrm{MCl}$, mild cognitive impairment.

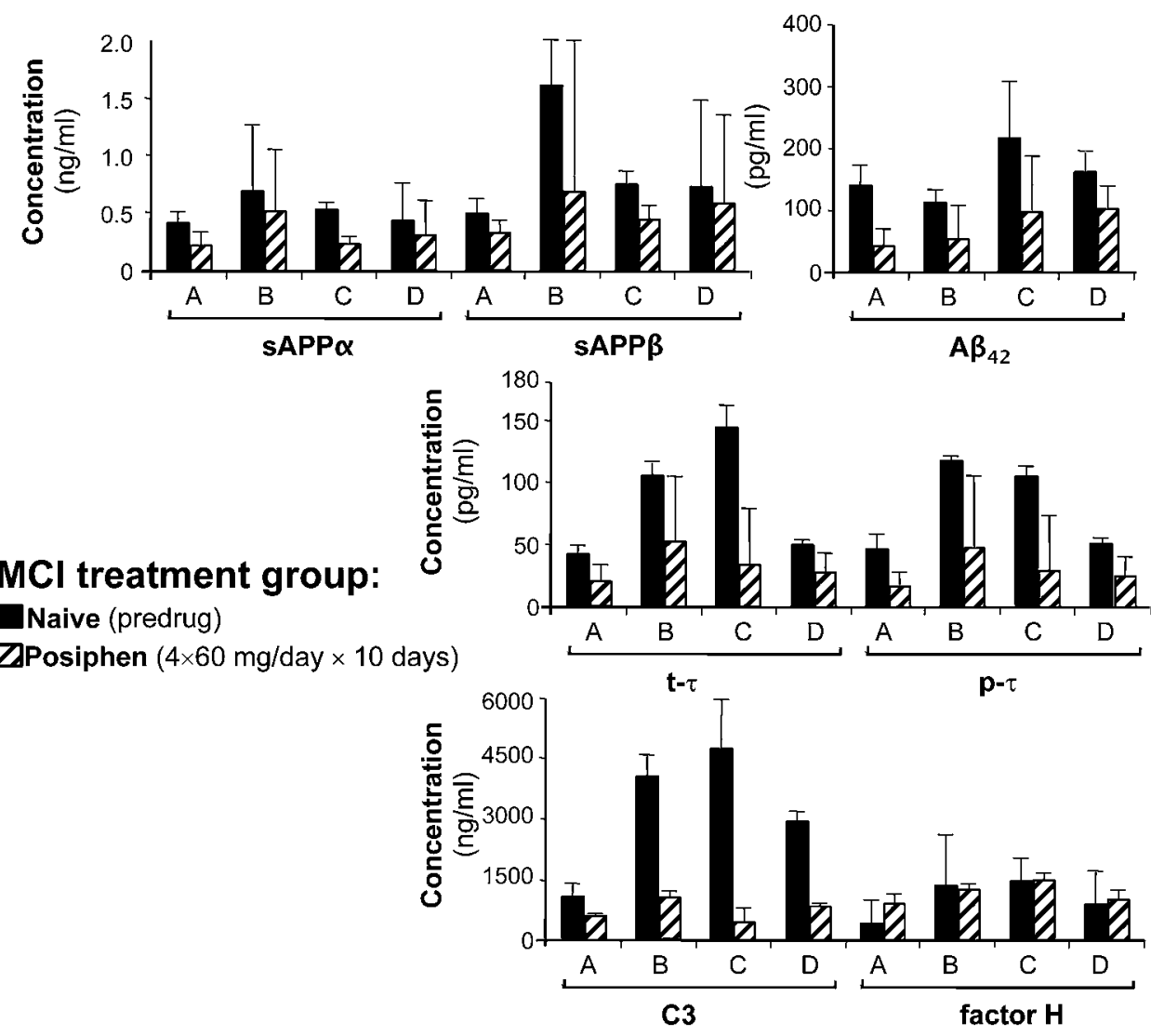


pattern of the changes was remarkably alike between the different assays employed blindly to measure the same CSF analyte at different independent institutions (whether AlphaLisa vs MSD in the quantification of $\operatorname{sPP} \alpha$ and $\mathrm{sAPP} \beta$, or AlphaLisa vs Innogenetics for $A \beta_{42}$ and $t-\tau$ ). Albeit, the percent of the Posiphen-induced inhibition and variance differed between the assay techniques (table 3); similar data deriving from the use of two independent assays provide a valuable level of cross-validation to help guard against unforeseen systematic errors. Clearly, without the potential to match predrug and postdrug time-dependent biomarker levels within the same patient, a far greater number of subjects would have been required to support statistical analyses. Nevertheless, a larger patient number, which is often limiting in early POM studies, would have provided greater statistical power to discriminate drug-induced biomarker actions, as would the inclusion of a placebo group.

Recent reports suggest that CSF elevations in SAPP $\alpha$ and, in particular, sAPP $\beta$ may be clinically useful and superior to assessing $A \beta_{42}$, in the early and differential diagnosis of incipient $A D{ }^{32}{ }^{33}$ Hence, as APP represents Posiphen's immediate target, CSF levels of sAPP $\alpha$ and sAPP $\beta$, rather than, simply, $A \beta_{42}$, were measured and found to be elevated in our MCI patients compared with healthy controls (figure 4), in accordance with others. ${ }^{33} 34$ Posiphen's reduction in CSF sAPP $\alpha$ and sAPP $\beta$ in MCI patients brought their values in line with healthy controls. A preliminary analysis of $A \beta_{42}$, analysed by two techniques (table $3 A$ ), suggests reductions in the same order as SAPP $\alpha$ and sAPP $\beta$.

Posiphen treatment led to statistically significant reductions in CSF levels of other key $\mathrm{AD}$ biomarkers, in particular $\mathrm{t}-\tau$ and $\mathrm{p}-\tau$. As illustrated in figure 4 and in accordance with others, 3536 CSF $t-\tau$ levels were elevated in our MCI patients versus healthy controls ${ }^{3738}$ and were normalised by Posiphen. The relevance of these actions and mechanisms through which they are mediated are a focus of current studies. In this regard, resembling the action of Posiphen to impact the translational regulation of APP mRNA, ${ }^{14} 1528 \tau$ can also be regulated at the level of its RNA stability, ${ }^{39} 40$ potentially by Posiphen. Alternatively, reductions in $\tau$ may be secondary to other actions or a combination of primary effects on translational regulation and secondary actions. Nevertheless, similar Posiphen-induced time-dependent reductions in $\tau$ have recently been found in neuronal cell cultures and preclinical $\mathrm{AD}$ models (Sambamurti K, personal communication). Posiphen, likewise, induced statistical declines in $\mathrm{MCI}$ CSF C3, a pro-inflammatory factor reportedly elevated in both $\mathrm{AD}$ transgenic mice ${ }^{41}$ and the CSF of $\mathrm{AD}$ subjects, ${ }^{42}$ together with key biomarkers (MCP-1, YKL-40) of microglial activation. ${ }^{24} 26$ In contrast, Posiphen treatment did not alter levels of the innate immune response protein $\mathrm{sCD} 14$ or factor $\mathrm{H}$. Likewise, the decline in specific inflammatory markers may be a secondary effect to the described reductions of APP and $\tau$.

In synopsis, our pharmacokinetic studies in humans and rodents permitted us to estimate levels of Posiphen/metabolites in human brain after Posiphen $(4 \times 60 \mathrm{mg} /$ day, 10 days) to be in the order of $3.5 \mu \mathrm{M}$ Posiphen, associated with the described biomarker changes. This drug level is greater than the determined $50 \%$ effective concentration of Posiphen to lower APP levels in neuronal cultures. ${ }^{13}$ Recent studies have demonstrated that each Posiphen metabolite, likewise, has APP lowering actions. ${ }^{43}$ We conclude that Posiphen appears to be a promising experimental drug for $\mathrm{MCI}$ and $\mathrm{AD}$ as it can effectively lower CSF levels of APP, its primary target in brain, and in addition lower $\mathrm{t}-\tau, \mathrm{p}-\tau$ and key inflammatory markers, and may hence impact disease progression at a number of levels.
Acknowledgements The authors are grateful to the following: (i) Qian-sheng Yu, Intramural Research Program, National Institute on Aging, NIH, for synthesis and chemical characterisation of highly pure samples of Posiphen, N1-norposiphen, N8norposiphen, and N1, N8-bisnorposiphen that were used as standards for analytical chemistry in pharmacokinetic studies. (ii) Harold W Holloway, Intramural Research Program, National Institute on Aging, NIH, for aid with pharmacokinetic studies. (iii) Karen Poksay and Olivier Descamps, Buck Institute for Research on Aging, for performing AlphaLisa analyses of CSF samples and (iv) David Fleet, Data Magik, for statistical analyses.

Contributors MLM contributed to the conception and design of the study, oversaw analyses (both experimental and statistical) and provided input into manuscript writing. MYC undertook experimental studies and analyses associated with CSF $S A P P \alpha$ and $S A P P \beta$ quantification. CP undertook experimental studies and analyses associated with CSF $A \beta, p-\tau, t-\tau, C 3$ and factor $H$. VJ aided in study design, was responsible for assays related to CSF A $\beta$, sAPP $\alpha$ and SAPP $\beta$ quantification, and provided input into manuscript writing. $\mathrm{HZ}$ aided in study design, was responsible for assays related to CSF MCP-1, YKL-40 and SCD14, and provided input into manuscript writing. NHG contributed to the study conception and design, the generation of Posiphen and metabolites, rodent studies, and the manuscript.

Funding This work was supported in part by QR Pharma, Inc. Henrik Zetterberg was supported by the Swedish Research Council (grant numbers K2010-63P-21562-01-4 and K2011-61X-20401-05-6) and the Swedish State Support for Clinical Research. Nigel H Greig was supported by the Intramural Research Program, National Institute on Aging, $\mathrm{NIH}$

Competing interests MLM and MYC are employees of QR Pharma, Inc. NHG is an inventor on the original Posiphen patent. Having assigned all rights to the US government, he declares that he has no ownership, financial interest or any other competing interests. All other authors declare no competing interests.

Ethics approval The human studies were conducted at and approved by the IRB and Ethics Committees of CEDRA/World Wide Clinical Trials (King of Prussia, PA) and the PRACS Institute (East Grand Forks, MN).

Provenance and peer review Not commissioned; externally peer reviewed.

\section{REFERENCES}

1. Stone JG, Casadesus G, Gustaw-Rothenberg K, et al. Frontiers in Alzheimer's disease therapeutics. Ther Adv Chronic Dis 2011;2:9-23.

2. Sambamurti $\mathbf{K}$, Greig NH, Lahiri DK. Advances in the cellular and molecular biology of the beta-amyloid protein in Alzheimer's disease. Neuromolecular Med 2002;1:1-31

3. Sambamurti K, Suram A, Venugopal C, et al. A partial failure of membrane protein turnover may cause Alzheimer's disease: a new hypothesis. Curr Alzheimer Res 2006:3:81-90.

4. Selkoe DJ. Alzheimer's disease. Cold Spring Harb Perspect Biol 2011;3:pii: a004457.

5. Popp J, Arlt S. Pharmacological treatment of dementia and mild cognitive impairment due to Alzheimer's disease. Curr Opin Psychiatry 2011;24:556-61.

6. Selkoe DJ. Defining molecular targets to prevent Alzheimer disease. Arch Neurol 2005:62:192-5.

7. Ashe KH, Zahs KR. Probing the biology of Alzheimer's disease in mice. Neuron 2010;66:631-45

8. De Felice FG, Velasco PT, Lambert MP, et al. A $\beta$ oligomers induce neuronal oxidative stress through an $\mathrm{N}$-methyl-D-aspartate receptor-dependent mechanism that is blocked by the Alzheimer drug memantine. J Biol Chem 2007:282:11590-601

9. Shankar GM, Li S, Mehta TH, et al. Amyloid-beta protein dimers isolated directly from Alzheimer's brains impair synaptic plasticity and memory. Nat Med 2008:14:837-42.

10. Nikolaev A, McLaughlin T, O'Leary DD, et al. APP binds DR6 to trigger axon pruning and neuron death via distinct caspases. Nature 2009:457:981-9.

11. Galvan V, Gorostiza OF, Banwait S, et al. Reversal of Alzheimer's-like pathology and behavior in human APP transgenic mice by mutation of Asp664. Proc Natl Acad Sci U $S$ A 2006;102:7130-5.

12. Harris JA, Devidze N, Halabisky B, et al. Many neuronal and behavioral impairments in transgenic mouse models of Alzheimer's disease are independent of caspase cleavage of the amyloid precursor protein. J Neurosci 2010;30:372-81.

13. Lahiri DK, Chen D, Maloney B, et al. The experimental Alzheimer's disease drug Posiphen lowers $A \beta$ peptide levels in cell culture and mice. J Pharmacol Exp Ther 2007;320:386-96.

14. Shaw K, Utsuki T, Rogers JT, et al. Phenserine regulates translation of ß-amyloid precursor protein mRNA by a putative interleukin-1 responsive element: a novel target for drug development. Proc Natl Acad Sci U S A 2001;98:7605-10.

15. Rogers JT, Randall JD, Cahill CM, et al. An iron-responsive element type II in the $5^{\prime}$ untranslated region of the Alzheimer's amyloid precursor protein transcript. J Biol Chem 2002;277:45518-28.

16. Cho HH, Cahill CM, Vanderburg CR, et al. Selective translational control of the Alzheimer amyloid precursor protein transcript by iron regulatory protein-1. J Biol Chem 2010;285:31217-32. 
17. Greig NH, Sambamurti K, Yu OS, et al. An overview of phenserine tartrate, a novel acetylcholinesterase inhibitor for the treatment of Alzheimer's disease. Curr Alzheimer Res 2005;2:281-90.

18. Marutle A, Ohmitsu M, Nilbratt M, et al. Modulation of human neural stem cell differentiation in Alzheimer (APP23) transgenic mice by phenserine. Proc Natl Acad Sci U S A 2007:104:12506-11.

19. Yu QS, Greig NH, Holloway HW, et al. Syntheses and anticholinesterase activities of (3aS)-N(1), N(8)-bisnorphenserine(3aS)-N(1), N(8)-bisnorphysostigmine, their antipodal isomers, and other potential metabolites of phenserine. J Med Chem 1998;41:2371-9.

20. Yu QS, Pei XF, Holloway HW, et al. Total syntheses and anticholinesterase activities of (3aS)-N(8)-norphysostigmine, (3aS)-N(8)-norphenserine, their antipodal isomers, and other N(8)-substituted analogues. J Med Chem 1997;40:2895-901.

21. Soares $H$, Raha N, Sikpi M, et al. A $\beta$ variability and effects of $\gamma$-secretase inhibition on plasma and CSF levels of $A \beta$ in healthy volunteers. Poster P1-264. International Conference on Alzheimer's Disease, Vienna, Austria: Alzheimer's Association, 2009.

22. Ereshefsky L, Jhee S, Yen M, et al. Cerebrospinal fluid beta-amyloid and dynabridging in Alzheimer's disease drug development. Biomark Med 2009;3:711-21.

23. Petersen RC. Mild cognitive impairment as a diagnostic entity. J Intern Med 2004:256:183-94.

24. Mattsson N, Tabatabaei S, Johansson P, et al. Cerebrospinal fluid microglial markers in Alzheimer's disease: elevated chitotriosidase activity but lack of diagnostic utility. Neuromolecular Med 2011;13:151-9.

25. Hong Z, Shi M, Chung KA, et al. DJ-1 and alpha-synuclein in human cerebrospinal fluid as biomarkers of Parkinson's disease. Brain 2010;133:713-26.

26. Yin GN, Jeon $\mathrm{H}$, Lee $\mathrm{S}$, et al. Role of soluble CD14 in cerebrospinal fluid as a regulator of glial functions. J Neurosci Res 2009;87:2578-90.

27. Salehi M, Faizi R, Takimoto J, et al. Examining the effects of $(+)$-phenserine in Ts65Dn mouse model of Down syndrome. Alzheimers Dement 2008;4:T462-3.

28. Rogers JT, Mikkilineni S, Cantuti-Castelvetri I, et al. The alpha-synuclein 5'untranslated region targeted translation blockers: anti-alpha synuclein efficacy of cardiac glycosides and Posiphen. J Neural Transm. 2011;118:493-507.

29. Bacchetti P, Deeks SG, McCune JM. Breaking free of sample size dogma to perform innovative translational research. Sci Transl Med 2011;3:87ps24.

30. Bateman RJ, Wen G, Morris JC, et al. Fluctuations of CSF amyloid-beta levels: implications for a diagnostic and therapeutic biomarker. Neurology 2007:68:666-9.
31. Huang Y, Potter R, Sigurdson W, et al. Effects of age and amyloid deposition on $a \beta$ dynamics in the human central nervous system. Arch Neurol 2012;69:51-8.

32. Lewczuk $\mathbf{P}$, Kamrowski-Kruck H, Peters 0 , et al. Soluble amyloid precursor proteins in the cerebrospinal fluid as novel potential biomarkers of Alzheimer's disease: a multicenter study. Mol Psychiatry 2010;15:138-45.

33. Perneczky R, Tsolakidou A, Arnold A, et al. CSF soluble amyloid precursor proteins in the diagnosis of incipient Alzheimer disease. Neurology 2011;77:35-8.

34. Alexopoulos P, Tsolakidou A, Roselli F, et al. Clinical and neurobiological correlates of soluble amyloid precursor proteins in the cerebrospinal fluid. Alzheimers Dement Published Online First: 4 November 2011.

35. Shaw LM, Korecka M, Clark CM, et al. Biomarkers of neurodegeneration for diagnosis and monitoring therapeutics. Nat Rev Drug Discov 2007:6:295-303

36. Seppälä TT, Koivisto AM, Hartikainen $\mathrm{P}$, et al. Longitudinal changes of CSF biomarkers in Alzheimer's disease. J Alzheimers Dis 2011;25:583-94.

37. Buchhave $\mathbf{P}$, Minthon L, Zetterberg $\mathrm{H}$, et al. Cerebrospinal fluid levels of $\beta$-amyloid $1-42$, but not of tau, are fully changed already 5 to 10 years before the onset of Alzheimer dementia. Arch Gen Psychiatry 2012;69:98-106.

38. Shaw LM, Vanderstichele H, Knapik-Czajka M, et al. Cerebrospinal fluid biomarke signature in Alzheimer's disease neuroimaging initiative subjects. Ann Neurol 2009;65:403-13.

39. Bottley A, Phillips NM, Webb TE, et al. elF4A inhibition allows translational regulation of mRNAs encoding proteins involved in Alzheimer's disease. PLoS One 2010;5:pii: e13030.

40. Veo BL, Krushel LA. Translation initiation of the human tau mRNA through an internal ribosomal entry site. J Alzheimers Dis 2009;16:271-5

41. Reichwald J, Danner S, Wiederhold KH, et al. Expression of complement system components during aging and amyloid deposition in APP transgenic mice. J Neuroinflammation 2009;6:35.

42. Wang $\mathrm{Y}$, Hancock AM, Bradner J, et al. Complement 3 and factor $\mathrm{h}$ in human cerebrospinal fluid in Parkinson's disease, Alzheimer's disease, and multiple-system atrophy. Am J Pathol 2011;178:1509-16.

43. Mikkilineni S, Cantuti-Castelvetri I, Cahill C et al. The anti-cholinesterase phenserine and its enantiomer Posiphen as $5^{\prime}$ untranslated region directed translation blockers of the Parkinson's alpha synuclein expression. Parkinson's Dis 2012:2012:142372. 